Strains of TRNG used in this study

\begin{tabular}{rlllll}
\hline Strain & Isolation date & Source & MIC $(\mathrm{mg} / \mathrm{l})$ & Disc diffusion test ${ }^{\star}(\mathrm{mm})$ & tetM type \\
\hline 60061 & 1985 & Yokohama & 16 & $15 \cdot 0$ & American \\
5120 & 1991 & Thailand & 64 & $10 \cdot 0$ & Dutch \\
6010 & 1994 & - & 32 & $12 \cdot 0$ & Dutch \\
\hline
\end{tabular}

${ }^{\star}$ A zone of inhibition from the edge of the tetracycline disc to the edge of confluent growth.

cline disc to the edge of confluent growth (table). In this study, we further characterised the tetM genes of these TRNG strains.

Ison et $a l^{1}$ previously reported the primer pair (A: 5'-GGCGTACAAGCACAAACTCG-3' and B: 5'-TCTCTGTTCAGGTTTACTCG-3') for detection of tet $M$ in $N$ gonorrhoeae. These sequences were derived from that of the Ureaplasma urealyticum tetM gene. ${ }^{2}$ More recently, the nucleotide sequences of the tetM genes of American and Dutch type plasmids have been determined and suggested that the tet $M$ determinant found in the American type plasmid has a different origin from that in the Dutch type. ${ }^{3}$ Because the base sequence of the tetM gene from Dutch type plasmid which corresponds to the primer $B$ is different from that of American type plasmid, ${ }^{3}$ primer B2 (5'-CCTTTGTTGAGGTTTGCTCG-3') was used instead of the primer B to detect Dutch type tetM. The cells grown on a Kellogg's agar medium were lysed in $100 \mu \mathrm{l}$ of distilled water for 10 minutes at $94^{\circ} \mathrm{C}$. As a template, $5 \mu \mathrm{l}$ of lysate was added to a polymerase chain reaction (PCR) $\mathrm{mix}-$ ture. The PCR mixture contained $0.2 \mathrm{mM}$ (each) deoxynucleoside triphosphate, 50 pmol of each oligonucleotide primer, TaKaRa Taq DNA polymerase (TaKaRa Shuzo, Kyoto, Japan), and buffers provided by the manufacturer in a total volume of $50 \mu \mathrm{l}$. The mixture was overlaid with $50 \mu \mathrm{l}$ of mineral oil and heated in a DNA thermal cycler PJ-2000 (TaKaRa) for 25 cycles consisting of 45 seconds at $94^{\circ} \mathrm{C}$, 60 seconds at $58^{\circ} \mathrm{C}$, and 60 seconds at $72^{\circ} \mathrm{C}$.

PCR amplification using the primer pair of $A$ and $B$ gave a product of the predicted size of 765 base pairs (bp) from strains 60061 but not from 5120 or 6010 . On the other hand, the primer pair of A and B2 amplified a $765 \mathrm{bp}$ fragment from strains 5120 and 6010 but not from 60061 . The restriction digests using $M s p I$ of the PCR products of 60061 gave the predicted three fragments of 370,260 , and $140 \mathrm{bp}$. MspI digests of the PCR products of 5120 and 6010 generated three fragments of 540,140 , and $90 \mathrm{bp}$. The amplified products were sequenced using the ABI PRISM Dye Terminator Cycle Sequencing Ready Detection Kit (Perkin-Elmer Corp CT, USA) and the ABI 310 Genetic Analyzer (Perkin-Elmer Corp), and that from 60061, and 6010 and 5120 were identical to corresponding sequence of the tet $M$ gene from American and Dutch type plasmids, ${ }^{3}$ respectively.

It is of interest that the isolation rate of TRNG was quite low and both American and Dutch type tetM genes were found in Tokyo and Kanagawa, Japan during the study period. A patient with a strain 60061 , isolated in 1985 was infected in Japan. A strain 5120 was imported from Thailand (table). These facts imply that TRNG already existed in 1985 in Japan and has been transported from other countries, but has not spread in Tokyo and Kanagawa area. Ison $e t a l^{1}$ found two types of HpaII (MspI) digestion pattern of the PCR products from tetM in TRNG strains. In this study we clearly distinguished the American type tetM gene from the Dutch type one using the PCR with the sets of the primer pairs. Further investigations will be needed to elucidate the prevalence of each type of tet $M$ gene in $N$ gonorrhoeae infections.

\section{T KUROKI} T MURASE

Y WATANABE

Y ASAI

S YAMAI

Department of Bacteriology and Pathology, Kanagawa Prefectural Public Health Laboratory, Nakao 1-1-1, Asahi-ku, Yokohama 241, Fapan S NAKAYAMA

H WATANABE Department of Bacteriology,

National Institute of Infectious Diseases, Toyama 1-23-1, Shinjyuku,

Tokyo 162, Japan

1 Ison CA, Tekki N, Gill MJ. Detection of the tetM determinant in Neisseria gonorrhoeae. Sex tet $M$ determinam Dis 1993;20:329-33.

2 Sanchez-Pescador $R$, Brown JT, Roberts $M$ Urdea MS. The nucleotide sequence of the tetracycline resistance determinant tet $M$ from Ureaplasma urealyticum. Nucleic Acids Res 1988;16:1216-7.

3 Gascoyne-Binzi DM, Heritage J, Hawkey PM Nucleotide sequences of the tet $(M)$ genes from the American and Dutch type tetracycline resistance plasmids of Neisseria gonorrhoea.

Accepted for publication 15 July 1997

\section{MATTERS ARISING}

\section{Epidemiology of genital Chlamydia tra-} chomatis

Simms et $\mathrm{al}^{1}$ in their review of the epidemiology of genital Chlamydia trachomatis in England and Wales state that "ad hoc prevalence and case finding studies carried out over the past 20 years were critically assessed in terms of study design and testing methodologies". The authors, however, do not define what is meant by "ad hoc" and do not make explicit how the cited literature was obtained, sifted, and appraised. As a consequence they fail to identify all relevant published prevalence studies.

I recently reviewed the literature relating to the prevalence of $C$ trachomatis infection in women attending British general practice $^{2}$ (which updated an earlier review of the literature ${ }^{3}$ ) and British family planning clinics. ${ }^{4}$ As I wished to ensure that all relevant studies were included I used an explicit search strategy and stated the reasons for inclusion or exclusion of the identified studies. This led to 15 relevant prevalence studies being identified and appraised. Simms $e t$ $a l,{ }^{1}$ in contrast, identified only six of these studies.

As far as general practice was concerned nine studies which met defined criteria were included in the review. It was concluded that the best current estimate of the prevalence of genital chlamydia in women attending general practice is $3 \%$ to $4 \%$ (estimates range from $2 \%$ to $12 \%$ ). ${ }^{2}$ This conclusion was based on the results of two large general practice prevalence studies ${ }^{56}$ which were not quoted by Simms et al. ${ }^{1}$ Six studies were identified from a review of the family planning literature with estimated prevalences of women attending family planning clinics ranging from $3 \%$ to $7 \%{ }^{4}$ The methodological quality of prevalence studies of genital chlamydia infection in women in general practice and at family planning clinics is, however, unsatisfactory. Common features of the studies reviewed were non-random sampling, small sample sizes, unclear inclusion/exclusion criteria, and use of different testing methods. This conclusion is in agreement with that of Simms et al. ${ }^{1}$

The discrepancy between my findings and those of Simms et al ${ }^{1}$ supports the argument made by the Evidence-Based Medicine Working Group that all reviews of the medical literature should make explicit how the cited literature was obtained, sifted, and appraised. ${ }^{7}$ Failure to do so is likely to lead to important papers being missed.

TIM STOKES

Department of General Practice and Primary Health Care,

University of Leicester, Faculty of Medicine, Leicester LE5 4PW

1 Simms I, Catchpole M, Brugha R, Rogers P, Mallinson H, Nicoll A. Epidemiology of genital Chlamydia trachomatis in England and Wales. Genitourin Med 1997;73:122-6.

2 Stokes T. Screening for Chlamydia in general practice: a literature review and summary of the evidence. I Publ Hith Med 1997;19: 222-32.

3 Oakeshott P, Hay P. General practice update: chlamydia infection in women. Brf Gen Pract 1995;45:615-20

4 Stokes T. Chlamydial infection in UK family planning clinics. Br f Fam Planning 1997 (in press)

5 Oakeshott P. Sexual health in teenagers. Lancet 1995;346:648-9.

6 Clay JC, Bowman CA. Controlling chlamydia infection. Genitourin Med 1996;72:145.

7 Oxman AD, Cook DJ, Guyatt GH. Users' guides to the medical literature. VI. How to use an overview. $\mathcal{F A M A} 1994 ; 272: 1367-71$.

\section{Reply}

Dr Stokes raises a number of important issues and correctly points out that the method of assessing the literature was not $N$ fully explained.' The paper aimed to provide a concise, comprehensive, and timely review of what is a rapidly expanding field. A literature search was carried out using Medline and by trawling the literature. The study was $c$ confined to England and Wales (one review cited by Dr Stokes was concerned with the United Kingdom as a whole). ${ }^{2}$ To ensure comparability between studies each paper was assessed in terms of the population studied, the presentation of results, and the $\mathbb{D}$ testing strategy used. Studies were only included where the raw data were presented for individual clinical settings. Chlamydial 8 diagnostic tests have undergone a rapid evolution over recent years and a wide variety of tests and testing strategies have been used. It is difficult, if not impossible, to make comparisons between all testing strategies but we decided to include only those studies that used a strategy based on screening and confirmatory tests.

Studies undertaken by physicians, micro-

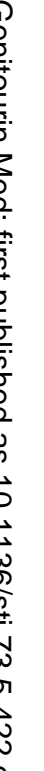

